Article

\title{
Discrepancy Analysis for Detecting Candidate Parcels Requiring Update of Land Category in Cadastral Map Using Hyperspectral UAV Images: A Case Study in Jeonju, South Korea
}

\author{
Seula Park and Ahram Song *(1) \\ Department of Civil and Environmental Engineering, Seoul National University, 1 Gwanak-ro, Gwanak-gu, \\ Seoul 08826, Korea; seula90@snu.ac.kr \\ * Correspondence: aram200@snu.ac.kr
}

Received: 6 December 2019; Accepted: 20 January 2020; Published: 21 January 2020

check for updates

\begin{abstract}
The non-spatial information of cadastral maps must be repeatedly updated to monitor recent changes in land property and to detect illegal land registrations by tax evaders. Since non-spatial information, such as land category, is usually updated by field-based surveys, it is time-consuming and only a limited area can be updated at a time. Although land categories can be updated by remote sensing techniques, the update is typically performed through manual analysis, namely through a visually interpreted comparison between the newly generated land information and the existing cadastral maps. A cost-effective, fast alternative to the current surveying methods would improve the efficiency of land management. For this purpose, the present study analyzes the discrepancy between the existing cadastral map and the actual land use. Our proposed method operates in two steps. First, an up-to-date land cover map is generated from hyperspectral unmanned aerial vehicle (UAV) images. These images are effectively classified by a hybrid two- and three-dimensional convolutional neural network. Second, a discrepancy map, which contains the ratio of the area that is being used differently from the registered land use in each parcel, is constructed through a three-stage inconsistency comparison. As a case study, the proposed method was evaluated using hyperspectral UAV images acquired at two sites of Jeonju in South Korea. The overall classification accuracies of six land classes at Sites 1 and 2 were $99.93 \%$ and $99.75 \%$ and those at Sites 1 and 2 are $39.4 \%$ and $34.4 \%$, respectively, which had discrepancy ratios of $50 \%$ or higher. Finally, discrepancy maps between the land cover maps and existing cadastral maps were generated and visualized. The method automatically reveals the inconsistent parcels requiring updates of their land category. Although the performance of the proposed method depends on the classification results obtained from UAV imagery, the method allows a flexible modification of the matching criteria between the land categories and land coverage. Therefore, it is generalizable to various cadastral systems and the discrepancy ratios will provide practical information and significantly reduce the time and effort for land monitoring and field surveying.
\end{abstract}

Keywords: Cadastral map; hyperspectral UAV; land category; land cover; land use; deep learning

\section{Introduction}

Cadastral maps show the boundaries and ownership of land parcels that separate adjacent land plots. These maps contain spatial information, such as shape, size, boundary, and location, as well as non-spatial information, such as land use, value, and tenure, which are uniquely encoded in textural or attribute files [1]. Moreover, cadastral maps are available as large-scale base maps with micro-level mapping [2]. As cadastral maps are related to personal properties, accurate cadastral mapping can 
improve agricultural productivity and support the national development policy [3]. Moreover, a well-structured cadastral map is a prerequisite for improved land management services [4].

A cadastral map is updated by modifying the spatial and non-spatial data of the existing cadastral maps to reflect the latest land information. High-quality cadastral mapping requires updating the changes in land use information and the spatial division of property units [5]. The land use type, which indicates the purpose of use, is registered and managed as an attribute of "land category" in a cadastral system. Therefore, the items of land category can be assigned according to their land use type, such as "Building site," "Parking lot," and "Road." Cadastral map updates are essential for not only recording the most recent land ownership and property division changes in a timely manner but also effectively managing the land information. For example, updating is necessary when the land is suddenly changed by new sub-divisions, transfer of land use, and natural disasters [6]. Furthermore, from the aspect of tax imposition, which is a main purpose of land use management by cadastral mapping [7], updating cadastral maps is crucial because the tax imposed on land owners depends on their land use type. Frequent updates of cadastral information can better manage illegal land use, whereby landowners register false land uses to reduce their taxes.

The procedure of updating cadastral maps can be divided into three steps: (1) extracting meaningful features and generating new data, (2) comparing new data with the existing base map and detecting changes, and (3) updating the base map with those changes and verifying the consistency of the updated map and actual information [8-10]. As the step of extracting relevant features, both up-to-date spatial and non-spatial information, such as parcel boundaries and land category, can be generated. Traditionally, cadastral surveying is performed by field work, aerial monitoring, and satellite data acquisition [5]. Although field surveying acquires accurate land information, it is extremely time-consuming and requires well-trained manpower for wide-area implementation. Remote sensing (RS) can be an effective alternative to field work because it is cheaper and faster compared to conventional cadastral surveys [3], and it is a useful data source for many base map-updating activities [8]. Cadastral boundaries set by roads, building, and water are visible in RS images and can be mapped from them.

To consider both generation of cadastral information and the further step of updating, which include a comparison between the generated information and existing cadastral maps, an integrated method that improves the efficiency of cadastral mapping and updating was proposed [5]. Using three bands of QuickBird satellite data, a digital and elevation model, and global navigation satellite system (GNSS) data, this method registers fused images to the existing cadastral map. After superimposing the boundaries of the cadastral map on the fused images, the map is updated by visual interpretation using a participatory geographic information system. Furthermore, the cadastral image was updated using CARTOSAT-2 panchromatic satellite images with $1.0 \mathrm{~m}$ resolution and Geo-eye multispectral images with GNSS data and $0.5 \mathrm{~m}$ resolution [6]. In this study, cadastral maps were updated by extracting the parcels from those images, along with three parameters (area, perimeter, and position) related to spatial elements, while non-spatial elements were not considered. Wassie et al. [11] proposed a procedure for extracting cadastral boundary information by semi-automatically using the WorldView-2 satellite data. In this study, the procedure of comparing the extracted information with the existing one, which is reference digital parcel boundaries, was performed in two ways: visual interpretation and quantitative analysis. The recentness of the information was not considered during the comparison, because this study aimed at verifying the accuracy of the extracted information rather than change detection for updating. Furthermore, the proposed procedure only focused on the parcel boundary, which comprises spatial elements.

Several previous studies on cadastral mapping and updating dealt with non-spatial updates, but focused only on extracting up-to-date information from the source date. The comparing and detecting changes step of updates were dependent on visual interpretation or performed in a limited range [2,9,10,12-14]. Specifically, Khadanga et al. [14] classified land use in cadastral parcels extracted from high-resolution satellite imagery through object-based image analysis (OBIA). The result layer of 
OBIA was written into a shapefile and compared with a digitized map of the cadastral parcels. The digitizing was manually performed and the comparisons were visually analyzed. Avramović et al. [12] updated the status of rural land use only from digital cadastral maps. Although they compared the land category items in the cadastral map with those in the real estate cadaster, they did not provide the details of the comparison.

To automate the comparison between the newly generated and existing cadastral information, the authors of [15] suggested a map-query-based comparison between the cadastral map and the land-cover map from satellite images. They generated the land cover map from Landsat TM satellite images and matched the land cover classes with the land category items in South Korea [15]. However, the spatial resolution of satellite images is relatively high $(30 \mathrm{~m})$ and obtaining images at the desired time is hindered by the time resolution and noise, such as cloud. After generating the land cover map, the authors performed a binary analysis of the pixel-level inconsistency between the land cover and cadastral maps. Although they analyzed heterogeneous data at the pixel-level, they calculated only the ratio of inconsistent area to the entire test area without considering the inconsistency by parcels or land category items. In conclusion, to improve the efficiency of the overall update process, it is vital to automate the comparison of up-to-date information with existing cadastral maps and the detection of parcel discrepancies.

When improving the efficiency of updating cadastral maps, one must consider the elaborateness of the latest cadastral information generated through various cadastral surveys. Although aerial and satellite surveying techniques acquire data over large-scale regions with superior spatial resolution, they are influenced by weather conditions, old acquisition time, and military security problems $[3,5]$. Therefore, unmanned aerial vehicles (UAVs) have recently been deployed for extracting up-to-date cadastral information. UAVs are cost-effective, especially in local applications, and acquire real-time data at high spatial resolution [10,16,17]. Manyoky et al. [18] noted that UAVs collect detailed information. Moreover, UAV-based methods enable an efficient documentation of the non-spatial information in cadastral maps, such as land use and vegetation. Relevant features are often extracted from orthophotos generated from UAV images using various feature-extraction methods such as image classification, segmentations, and line extraction [9]. As an example of cadastral mapping and updating with UAV imagery, areas subjected to landslides, which manifest as a sudden change in land use, were automatically detected from UAV images [19]. The detected changes in land use provided the basis information for synchronizing the cadastral information. However, the target area was manually extracted through an overlay analysis between the information extracted from the UAV and the cadastral map. Moreover, the updates were performed on limited target parcels (landslide areas) rather than the whole area. Manyoky et al. [18] compared the use of UAVs with the tachymeter-GNSS combined method in cadastral mapping and updating. The acquired points for generating and updating the cadastral maps were classified by land cover, such as vegetation types, buildings, and streets. However, the authors did not thoroughly describe the data processing steps for generating and updating the cadastral information.

As mentioned earlier, the land category as non-spatial data directly affects land value estimation and thus needs to be up-to-date in a short cycle. The inconsistency between the registered land category and actual land use when updating cadastral maps is a well-reported problem in cadastral mapping $[12,13,17,20]$. In South Korea, items of land category are determined based on the primary use of each parcel [21], which is directly related to the assessment value of the land [15]. Therefore, it is a legal obligation to correct the registration if the registered land information differs from the actual land use information [21]. New information can be updated by the land owner's registration. Fines or imprisonment may be imposed for those who do not inform about a change in land category or a false notification [21,22].

As the accuracy of an update is associated with ownership of property, the update must be verified through a field survey. This is especially important for updates of cadastral systems (including maps). Detecting the areas requiring update is crucial for reducing the target area of the field survey and 
improving the effectiveness of the field work. An automatic process would facilitate cadastral mapping and updating. To this end, the present study proposes a new discrepancy analysis method that automatically detects candidate parcels requiring an update of their land category information. The proposed method is implemented in two stages: generating up-to-date land category information and comparing the new information with the existing cadastral information. To effectively extract the land cover, we use hyperspectral UAV images and a deep learning approach. Hyperspectral UAV images can spectrally discriminate similar materials that cannot be identified in RGB or multi-spectral images captured at specific times. In the latter stage, the land category information generated from a UAV is compared with the existing cadastral map managed by the government. The comparison process generates a discrepancy map representing the parcels requiring update. The major contributions of the proposed method are stated below.

- For generating up-to-date land category information, we combine two-dimensional (2D) and three-dimensional (3D) convolutional neural networks (CNNs) to classify hyperspectral UAV images, and hence, generate the latest land category information at specific times and intervals. Furthermore, the environmental settings for learning are demonstrated and the classification results are analyzed to understand when the proposed network was applied to hyperspectral UAV images.

- For detecting discrepancies between the new information and the existing cadastral information, the efficiency of updating the registered land category is improved by a new technique that automatically compares two sets of non-spatial information under different criteria and structures.

The remainder of this paper is organized as follows. Section 2 proposes our discrepancy analysis method, and Section 3 describes the datasets, environmental conditions of the experiments, and the results of a case study in South Korea. Finally, the conclusions are provided in Section 4.

\section{Methods}

As shown in Figure 1, the proposed method of discrepancy analysis comprises two main parts: (i) classifying hyperspectral UAV images using the hybrid CNN for generating the land cover map and (ii) comparing the consistency between the cadastral map and land cover map for detecting inconsistent parcels through a query-based approach.

(1) The hybrid CNN with 2D and 3D kernels extracts the spatial-spectral features from hyperspectral UAV images and obtains a land cover map depicting the regions covered by forests, crops, bare soils, water, roads, and buildings. The images input to the hybrid CNN are pre-processed by principle component analysis (PCA) to reduce the number of redundant spectral bands and the computational cost. The hybrid $\mathrm{CNN}$ then classifies images by extracting various meaningful feature maps. The resulting land cover map provides the latest land information on sites.

(2) To the procedure that automatically detects inconsistent parcels, two maps are input: the existing cadastral map, which is managed by the government, and the land cover map, which is generated from hyperspectral UAV image classification. To compare the heterogeneous datasets with vector and raster structured data, the procedure adopts an encoding-decoding approach. The final output is a discrepancy map generated through query-based comparison of the mapping information in the land category items and the land cover classes in different frameworks. 


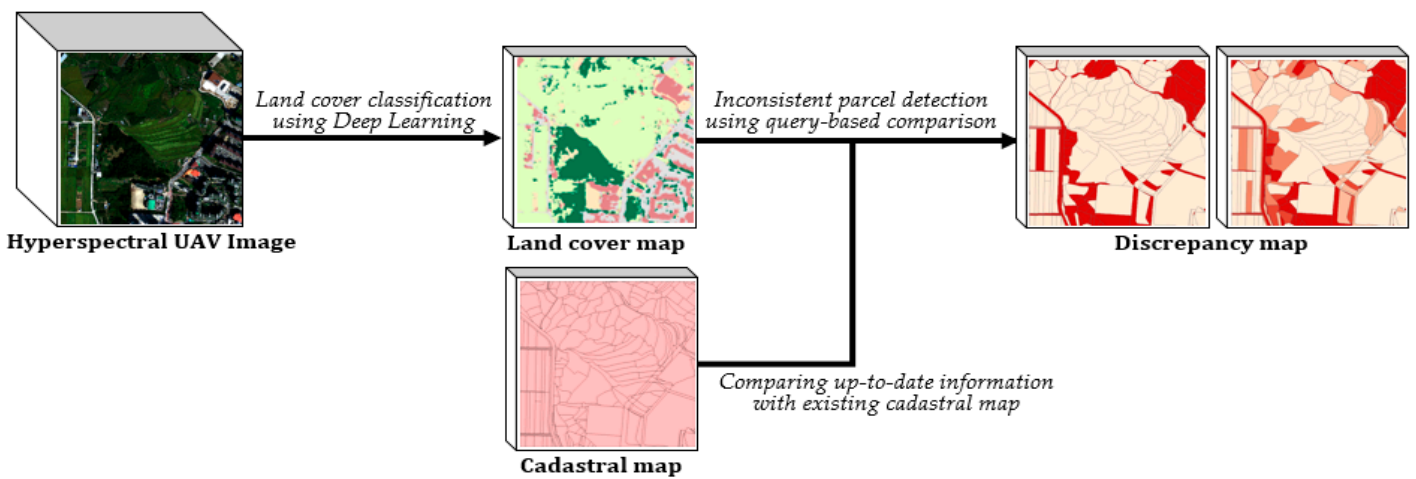

Figure 1. Proposed process of discrepancy analysis. UAV: unmanned aerial vehicle.

\subsection{Step 1: Hyperpsectral UAV Image Classification For Generating the Land Cover Map}

Hyperspectral images (HSIs) contain hundreds of narrow continuous bands over a wide range of the electromagnetic spectrum. Therefore, they provide more detailed spectral information than multispectral images and can spectrally discriminate similar materials. A land cover map derived from HSIs distinguishes distinct classes, such as forest and crop land, which are included in the land category framework. Level-I classes of land cover can be regarded as the usage information. In this sense, the land cover information from HSIs contains not only the land surface materials but also the land use. Furthermore, the latest land surface information of the target area can be extracted from UAV images taken at the desired time point in the desired interval.

HSI classification methods should consider the high dimensionality of the dataset. Traditionally, HSIs have been classified by pattern recognition algorithms, such as nearest neighbor, decision trees, and linear functions [23]. k-nearest neighbor (k-NN) clustering is a representative simple method that measures the similarities between the training and test data by using their Euclidean distances. Support vector machines remove the curse of dimensionality by determining the boundaries in a high-dimensional space, using the kernel method [23].

More recently, HSI classification has been performed by deep learning approaches. Deep learning replaces the hand-crafted feature-engineering process, which requires expert experience and careful parameter settings, with automatic extraction of the meaningful features contained in high-dimensional bands [24]. CNNs have been widely applied to HSI classification tasks [25-28]. Many studies have successively classified the items in hyperspectral images using 2D-CNNs, which extract features from spatial domains $[25,26]$. Efficient feature extraction by $2 \mathrm{D}-\mathrm{CNN}$ sequires a data transformation process, such as data reduction, to convolute all bands of the input image. As HSIs include hundreds of spectral bands, the convolutions require several kernels, which introduces the over-fitting problem and increases the computational cost. $2 \mathrm{D}$ convolution is computed as follows:

$$
v_{l, j}^{x, y}=\phi\left(\sum_{n} \sum_{h=0}^{H-1} \sum_{w=0}^{W-1} w_{l j n}^{h w} o_{(l-1) n}^{(x+h)(y+w)}+b\right),
$$

where $v_{l, j}^{x, y}$ is the pixel value of position $(x, y)$ on the $j$ th feature map in layer $l$ (the layer of the current operation); $\phi$ is the activation function; $b$ is a bias parameter; and $w_{l j n}^{h w}$ is the weight value at position $(h, w)$ in the $n$th shared $H \times W$ kernel, where $n$ is the number of feature maps in the $(l-1)$ th layer. $o_{(l-1) n}^{(x+h)(y+w)}$ is the input at position $(x+h)(y+w)$ and $(h, w)$ denotes its offset to $(x, y)$.

3D-CNNs simultaneously extract the spatial and spectral features [27,28]. A 3D-CNN preserves the original input data by avoiding complex data reconstruction and considers the relationships among channels; however, 3D-CNNs are more computationally complex than 2D-CNNs. In classes with 
similar textures over many spectral bands, they can perform worse than 2D-CNNs [29]. The pixel value at position $(x, y, z)$ in the jth $3 \mathrm{D}$ feature cube of the lth layer is given as follows:

$$
v_{l, j}^{x, y, z}=\phi\left(\sum_{n} \sum_{h=0}^{H-1} \sum_{w=0}^{W-1} \sum_{r=0}^{R-1} w_{l j n}^{h h w} o_{(l-1) n}^{(x+h)(y+w)(z+r)}+b\right),
$$

where $R$ is the spectral dimension of the $3 \mathrm{D}$ kernel and $w_{l j n}^{h w r}$ is the weight value at position $(h, w, r)$, connected to the $n$th feature in the $(l-1)$ th layer. $o_{(l-1) n}^{(x+h)(y+w)(z+r)}$ represents the input at position $(x+h)(y+w)(z+r)$ and $(h, w, r)$ denotes its offset to $(x, y, z)$.

The abovementioned limitations can be resolved by hybridizing 2D- and 3D-CNNs [29]. In the hybrid spectral CNN (HybridSN), the output of a 3D-CNN is input to a 2D-CNN. This configuration learns the spatial representation at a more abstract level, with lower model complexity, compared to the 3D-CNN alone. The present study proposes a new hybrid 2D-CNN and 3D-CNN for effectively classifying hyperspectral UAV images (Figure 2). The network comprises 2D- and 3D-CNN branches in convolutional layers, which generate various meaningful feature maps from the input. First, spectral redundancy is removed by PCA along the spectral bands of the original HSI. The PCA image is then processed through the convolutional layers with 2D and 3D kernels. The first convolutional layers of both branches have eight filters, and the subsequent convolutional layers of the $2 \mathrm{D}$ and $3 \mathrm{D}$ branches have 16 and 32 kernels, respectively. The outputs of the 3D convolutional layers are converted to a 2D shape and the feature maps obtained from both branches are combined to form the spectral and spatial feature maps. These maps are input to the fully connected layers. Finally, the pixels are classified into land cover classes. In the next process, these land cover classes are mapped to the land category items in the cadastral map. To reduce the complexity of the mapping and to generalize the model, we adopt level-I types of land cover, namely forests, crop lands, roads, buildings, bare soil, and water bodies.

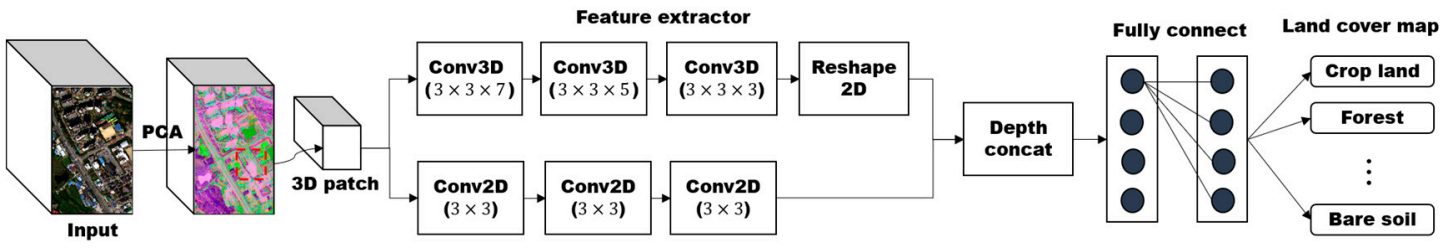

Figure 2. Process of step1 in discrepancy analysis: hyperspectral UAV image classification for generating the land cover map. PCA: principle component analysis.

\subsection{Step 2: Inonsistency Comparison Between the Cadastral Map and Land Cover Map}

Our proposed pixel-level inconsistency comparison automatically detects the areas of inconsistent land use between the registered and actual land information. In a previous study [30], a restructured land use map was generated in vector format, which assigned the actual land cover classes from the imagery as attributes and the cadastral boundary as the geometry. Although this map compares the registered land categories in cadastral maps with the actual land use, it is limited to the primary land use, which occupies the maximum area in each parcel. An elaborate comparison must consider all land uses in each parcel. Figure 3 shows the process of comparing the actual land cover and cadastral map at the pixel-level, which considers both minor and primary uses. 


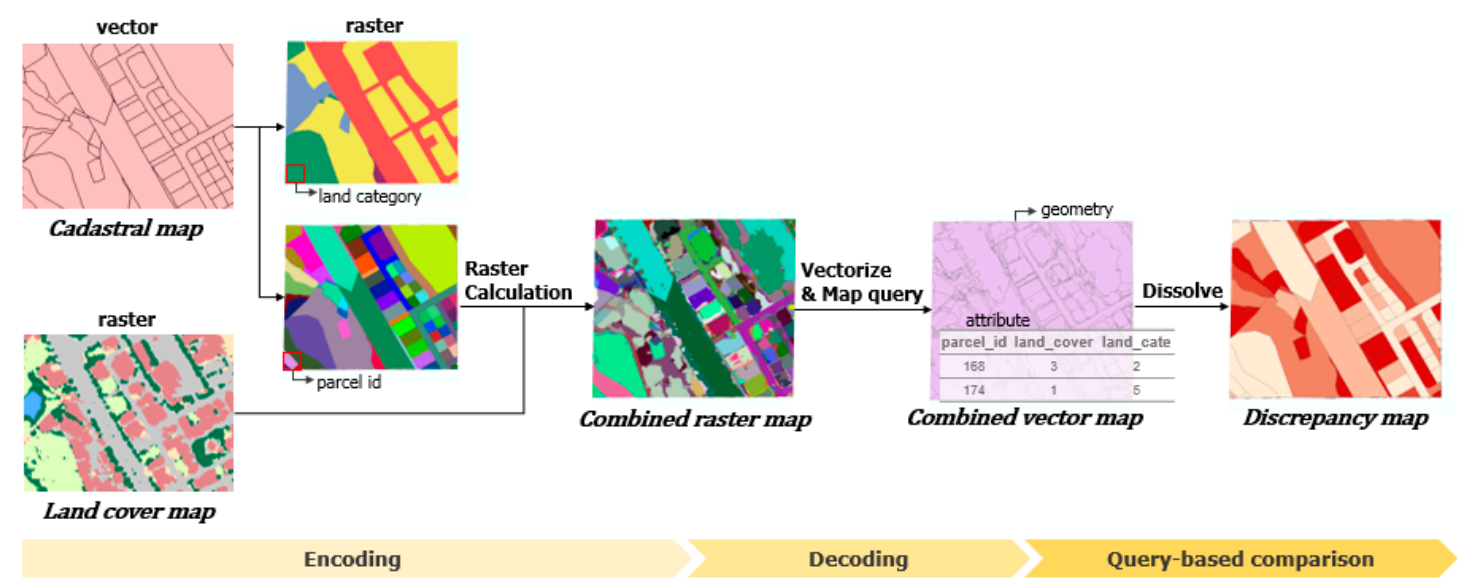

Figure 3. Process of step 2 in discrepancy analysis: inconsistency comparison between cadastral and land cover maps.

The proposed automatic comparison technique is then divided into three stages: "Encoding," "Decoding," and "Query-based comparison" (Figure 3). Because the cadastral map and land cover map are constructed in vector and raster formats, respectively, the automatic inconsistency comparison must convert the heterogeneous datasets into the same structure prior to the overlay analysis [15]. The first encoding step performs raster conversion using the cadastral map attributes. For this purpose, the land category and parcel ID are assigned to each pixel of the rasterized cadastral maps, which have the same pixel size as that of the land cover map. A combined raster map is then generated with coded values $V_{i j}$ combining the land cover $C_{i j}$, land category $U_{i j}$, and parcel ID $P_{i j}$ values. An encoding query is expressed as follows:

$$
V_{i j}=P_{i j} \times 10^{4}+U_{i j} \times 10^{2}+C_{i j} \forall(i, j) .
$$

The second decoding stage vectorizes the combined raster map, which results in a vector map combining the land cover and land category information. The attributes of the vector map include the parcel ID, land cover, and land category, and their values are assigned by decoding the pixel values.

The combined vector map includes both the land category values and land cover values in a unit area. Therefore, the inconsistent area can be automatically extracted through a query-based comparison between the corresponding values in the previous stage. The land category items are defined in terms of land use, and each item can contain multiple usages. For example, a "building site" may include buildings and bare land, and a "school site" may include buildings, bare land, trees, and grass. However, when extracting the land cover information from the imagery, the materials and/or objects covering the land are extracted from the spectral characteristics of the image. When constructing a query for comparing these two maps, we must define mapping rules that determine the discrepancy between the land category items and land cover classes, which are classified under different criteria. However, establishing an absolute standard for mapping land category items to land cover classes is restricted because the land category items differ among country-specific cadastral systems and the number of available classification classes depends on the quality of the imagery. In the case study (Section 4.1), the mapping between land cover classes and land category items is performed under the Korean Cadastral System as a guideline. An automatic comparison can be queried based on the corresponding mapping information; the query result can automatically determine the discrepancy between the land category and land cover. The discrepancy map can be generated by dissolving the area based on parcel IDs. From the discrepancy map, we can calculate the portions of inconsistent areas where the registered land category is different from the actual land cover in each parcel. Because the discrepancy map is generated by comparing both the primary and minor land uses, it provides reference data for the automatic detection of parcels that must be divided. Table 1 shows the proposed 
algorithm of a pixel-level comparison for detecting inconsistent areas; moreover, this algorithm can be automated in the model builder of ArcGIS 10.1 [31] (Figure 4).

Table 1. Proposed algorithm of pixel-level inconsistency comparison.

\begin{tabular}{|c|c|}
\hline Input: & $\begin{array}{l}\text { Land Cover Map (LC, Rraster) } \\
\text { Cadastral Map (CM, Vector) }\end{array}$ \\
\hline 1: & \# Encoding \\
\hline 2: & $\begin{array}{l}\text { R_Cate = rasterized CM by assigning values with "land } \\
\text { category" }\end{array}$ \\
\hline 3: & $\mathrm{R} \_\mathrm{PI}=$ rasterized CM by assigning values with "Parcel ID" \\
\hline 4: & $\mathrm{w}, \mathrm{h}=$ width, height (image extent) of LC \\
\hline 5: & CRM (Combined Raster Map) $=$ empty raster layer with $\mathrm{w} \times \mathrm{h}$ \\
\hline 6: & For each pixel $(i, j)$ on CRM: \\
\hline 7: & $P(i, j)=$ assigned value of pixel $(i, j)$ on $R \_P I$ \\
\hline 8: & $U(i, j)=$ assigned value of pixel $(i, j)$ on $R \_$Cate \\
\hline 9: & $C(i, j)=$ assigned value of pixel $(i, j)$ on LC \\
\hline 10: & Combined $(i, j)=P(i, j) \times 10^{4}+U(i, j) \times 10^{2}+C(i, j)$ \\
\hline 11: & assign value of Combined(i,j) on pixel $(\mathrm{i}, \mathrm{j})$ to generate CRM \\
\hline 12: & end \\
\hline \multicolumn{2}{|c|}{ Intermediate Output: Combined Raster Map (CRM, Raster) } \\
\hline 13: & \# Decoding \\
\hline 14: & CVM $($ Combined Vector Map $)=$ Raster to Polygon $(\mathrm{CRM})$ \\
\hline 15: & For each polygon i on CVM: \\
\hline 16: & $\mathrm{PV}(\mathrm{i})=$ pixel value of polygon $\mathrm{i}$ \\
\hline 17: & CVM(i).p_id $=$ PV(i) $/ 10^{4}$ \\
\hline 18: & CVM(i) category $=\left(\mathrm{PV}(\mathrm{i}) \% 10^{4}-\mathrm{PV}(\mathrm{i}) \% 10^{2}\right) / 10^{2}$ \\
\hline 19: & CVM(i).cover $=\mathrm{PV}(\mathrm{i}) \% 10^{2}$ \\
\hline 20: & CVM(i).area_ia, CVM(i).area_ca $=0$ \\
\hline 21: & end \\
\hline \multicolumn{2}{|c|}{ Intermediate Output: Combined Vector Map (CVM, Vector) } \\
\hline 22: & \# Query-based comparison \\
\hline 23: & $\begin{array}{l}\text { Make query } Q \text { using mapping information between land } \\
\text { category and land cover }\end{array}$ \\
\hline 24: & $\mathrm{TF}=$ Execute query $\mathrm{Q}$ on CVM \\
\hline 25: & If $\mathrm{TF}==$ true: \\
\hline 26: & CVM(i).area_ia = calculate area of polygon i \\
\hline 27: & else: \\
\hline 28: & CVM(i).area_ca $=$ calculate area of polygon $\mathrm{i}$ \\
\hline 29: & end \\
\hline 30: & $\begin{array}{l}\text { DM (Discrepancy Map) = Dissolve }(\mathrm{CVM}) \text { based on p_id with } \\
\text { summation of area_ia and area_ca }\end{array}$ \\
\hline 31: & For each polygon i on MDA: \\
\hline 32: & DM (i).ic_ratio= area_ia(i)/area(i) \\
\hline 33: & end \\
\hline \multicolumn{2}{|c|}{ Output: Discrepancy Map (DM, vector) } \\
\hline
\end{tabular}

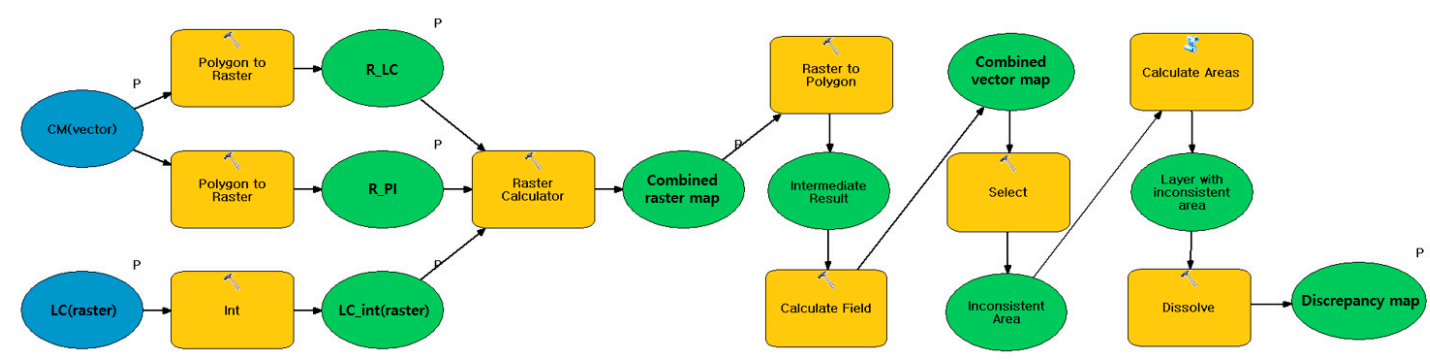

Figure 4. Automated model for detecting inconsistent area. 
The generated discrepancy map can be utilized for another purpose: detecting parcels requiring division. Specifically, because the cadastral map was created by assigning one land category value (based on the primary use) per parcel, parcels with a high ratio of minor-use area must be divided for efficient land management [21]. The proposed process reflects all uses of the land. Therefore, it automatically detects inconsistent areas while detecting the parcels that must be divided into different land use statuses.

\section{Dataset}

The dataset contained the hyperspectral UAV images acquired at two sites of Jeonju City in South Korea. The hyperspectral UAV images were acquired on September 19, 2019, by a DJI Matrice 200 UAV equipped with hyperspectral sensors (Corning microHSI SHARK 410). This platform had accurate flight controls and inherent stability. Its spatial resolution was $15 \mathrm{~cm}$ and spectral resolution was 4 nm over 150 bands ranging from 398.78 to $996.74 \mathrm{~nm}$. The flight path of the UAV was selected to follow the waypoint at a flight height of $200 \mathrm{~m}$. The whole study area $(890 \mathrm{~m} \times 730 \mathrm{~m})$ was covered in 15 courses. Study sites of area $600 \mathrm{~m} \times 600 \mathrm{~m}$, where the errors associated with camera shaking and gematric problems were few, were selected from the whole area. The images were registered using the geographic map projection WGS-84. The center coordinates of Sites 1 and 2 were $\left(35^{\circ} 48^{\prime} 19^{\prime \prime} \mathrm{N}\right.$, $\left.127^{\circ} 05^{\prime} 45^{\prime \prime} \mathrm{E}\right)$ and $\left(35^{\circ} 47^{\prime} 16^{\prime \prime} \mathrm{N}, 127^{\circ} 07^{\prime} 14^{\prime \prime} \mathrm{E}\right)$, respectively (Figure 5). These sites included crop lands, forests, and building areas. Owing to the high spatial resolution of the hyperspectral UAV images, objects such as vehicles, the centerlines of roads, and shadows, besides buildings and trees, could be identified. As such information was unnecessary for updating the cadastral map, the spatial resolution of the images was reduced to $60 \mathrm{~cm}$ to limit the number of classification classes and reduce the memory requirements of deep learning. Prior to the classification, the images were pre-processed by geometric and radiometric corrections based on GNSS and field spectrometry data.
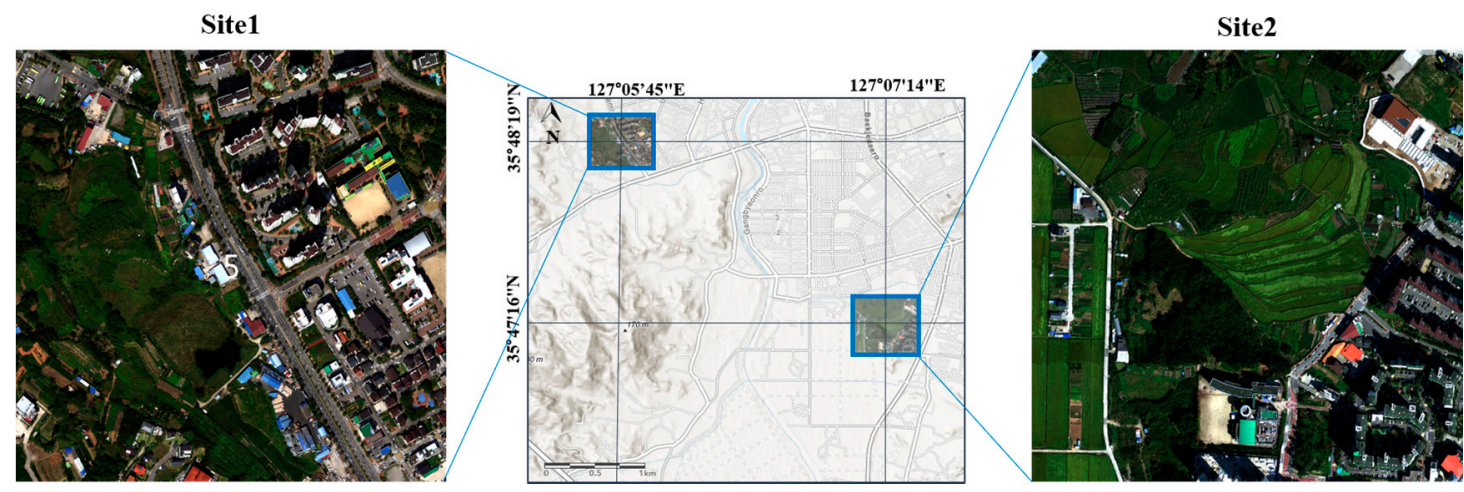

Figure 5. Locations of the two study sites in South Korea, along with their UAV hyperspectral images. The background map was obtained from ArcGIS (a geographic information system (GIS) for working with maps and geographic information maintained by Esri) world map [32]. The hyperspectral UAV images were obtained on 19 September 2019.

Figure 6 shows the cadastral maps of the study sites. There were 284 and 250 parcels in the cadastral maps of Sites 1 and 2, respectively. We obtained the most recently updated serial cadastral map taken in January 2018. In Korea, land categories of cadastral maps can be divided into 28 items, and a cadastral map can be divided into 28 main land categories. The study sites included 17 land category items: building sites, paddy fields, fields, park sites, school sites, roads, forests, reservoirs, miscellaneous land, sites for religious use, parking lots, ditches, factory sites, cemeteries, gas station sites, sports areas, and ranches. 


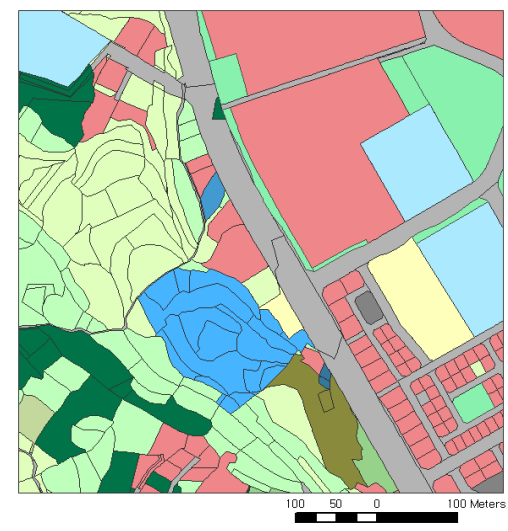

(a)

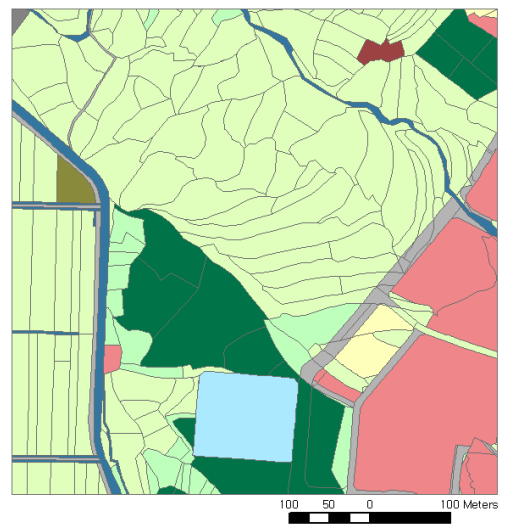

(b)

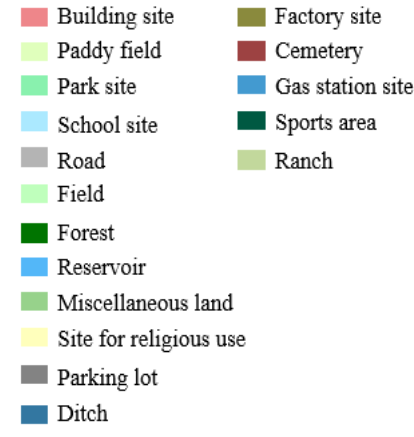

Figure 6. Cadastral map of the study sites in South Korea: (a) Site 1, (b) Site 2.

\section{Results}

\subsection{Classification Results}

The hyperspectral UAV images were classified by the proposed hybrid CNN. The network was optimized in 30 epochs of Adam with a learning rate of $10^{-3}$ and a batch size of 256 . The Adam optimizer is a combination of the stochastic gradient descent with momentum and RMSprop, and has relatively low memory requirements and is quite computationally efficient [33]. At the start of each iteration, the network was randomly initialized. The ground-truth data were manually defined from the field data. The field work acquired the spectral libraries and types of surface materials. The ground truth was composed of 88,567 pixels and contained six classes: crops, forests, roads, buildings, bare soil, and water. The classes that could be mapped to the land category items were then defined. The various crop lands and grass covers were combined into "crop land," and relatively high trees were classified as "forest." Colored roofs, such as blue, brown, and white, were all classified as "buildings." "Bare soil" represented ground without buildings and vegetation, and "road" encompassed asphalt roadways. The ground-truth data were randomly divided into training, validation, and test samples. Sixty percent of the ground-truth data (53,140 pixels) were used as training samples, which were subdivided into validation and training data at a ratio of 7:3 to avoid overfitting problems. The remaining $40 \%$ of the ground-truth data $(35,427$ pixels) were reserved as the test samples. The performance of the proposed network was estimated from the classification accuracy of the test data.

To confirm the effectiveness of the hybrid network, the classification accuracies of the 2D and 3D-CNNs were compared. Both networks were composed of three convolutional layers and used the same variables as those used by the hybrid CNN. In each experiment, the performance of the network was evaluated by the F1 scores of the six classes and the overall accuracy (OA). The F1 score measured the classification accuracy in terms of the precision and recall scores (Equation (4)). Precision defines the fraction of correctly retrieved instances among all instances, and recall is the fraction of correctly retrieved instances among all correct instances.

$$
\text { F1 score }=2 \times \frac{\text { Precision } \times \text { Recall }}{\text { Precision }+ \text { Recall }} \text {. }
$$

Figure 7 shows the classification losses and accuracies in each epoch for the training and validation samples of Sites 1 and 2. The hybrid CNN achieved lower classification loss and higher accuracy compared to 2D-CNN and 3D-CNN. Relative to 2D-CNN, the loss reduction and accuracy improvement in the hybrid CNN became more noticeable with increasing epoch number. Although 3D-CNN also achieved higher accuracy than 2D-CNN, it was less accurate and incurred higher losses than the hybrid 
$\mathrm{CNN}$ at Site 1. According to the results, 3D-CNN was more useful for classifying hyperspectral images than $2 \mathrm{D}-\mathrm{CNN}$ but combining the 2D and 3D CNNs improved the classification performance.

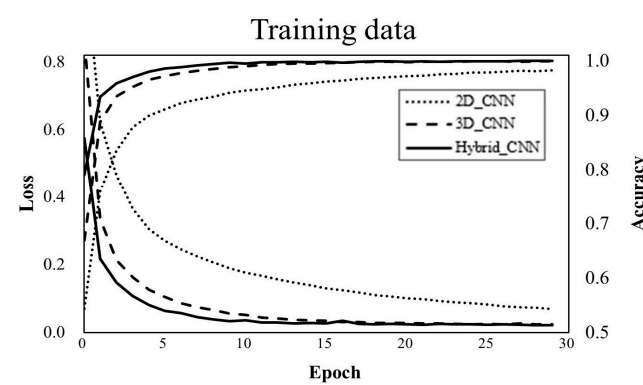

(a)

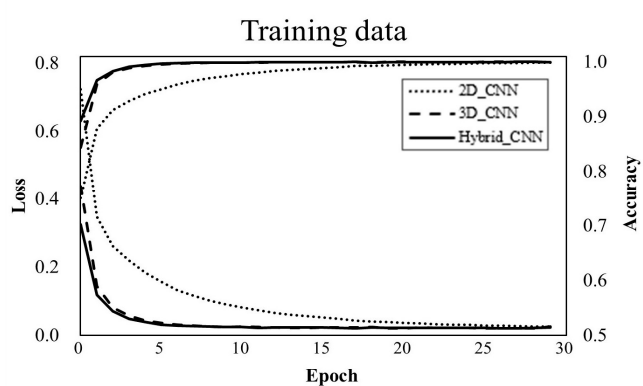

(c)

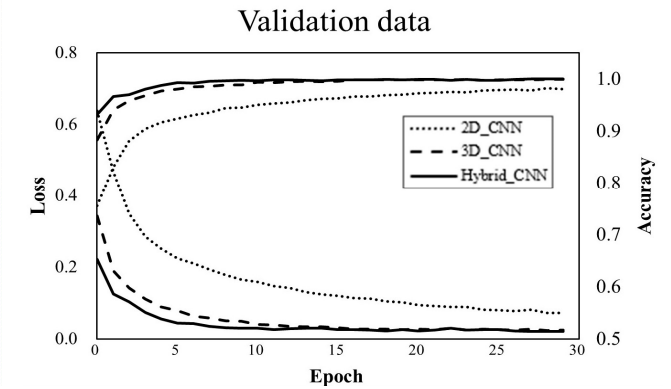

(b)

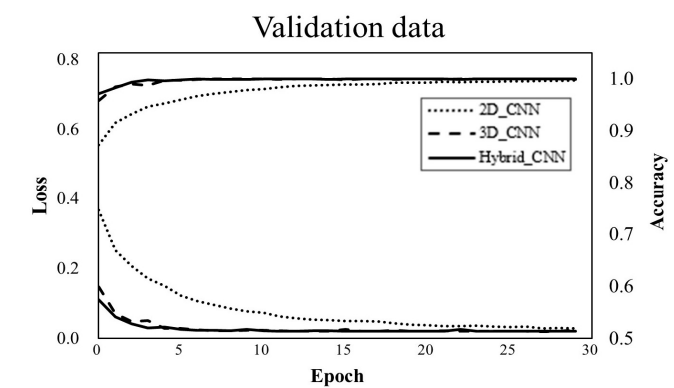

(d)

Figure 7. Classification losses and accuracies in each epoch on (a) training samples, (b) validation samples at Site 1, (c) training samples, (d) validation samples at Site 2.

Figure 8 shows the classification results of the hyperspectral UAV images using the hybrid CNN. The F1 scores and overall accuracies of the six classes are listed in Table 2. The OAs of the land cover classifications at Sites 1 and 2 were $99.93 \%$ and $99.75 \%$, respectively. Because the ground-truth data did not cover the entire study area, it was not the classification accuracy of the entire image but rather that of a randomly selected test sample location. According to Table 2, all six classes were well classified. As there was no water at Site 2, the results of this site were divided into five classes. Forests and roads obtained a lower F1 score than the other classes, because the spectral characteristics of crop land and forest were very similar. Furthermore, roads, parking lots, and car were classified into the "road" class and various colored rooves were classified into the "building" class. Moreover, areas that appeared to be farmland with low vegetation were classified as "bare soil." Pixel-level classification errors in the results can be considered as insignificant because an inconsistency comparison will be conducted at the parcel level.

Table 2. Classification results of the South Korean sites: F1 score and overall accuracy.

\begin{tabular}{cccccccc}
\hline & \multicolumn{9}{c}{ F1 Score } & \multirow{2}{*}{ OA (\%) } \\
\cline { 2 - 7 } & Crop Land & Forest & Road & Building & Water & Bare Soil & \\
\hline Site1 & 0.9998 & 0.9990 & 0.9993 & 0.9994 & 1.0000 & 0.9984 & $99.93 \pm 0.1$ \\
\hline Site2 & 0.9999 & 0.9935 & 1.0000 & 0.9984 & - & 0.9705 & $99.75 \pm 0.1$ \\
\hline
\end{tabular}




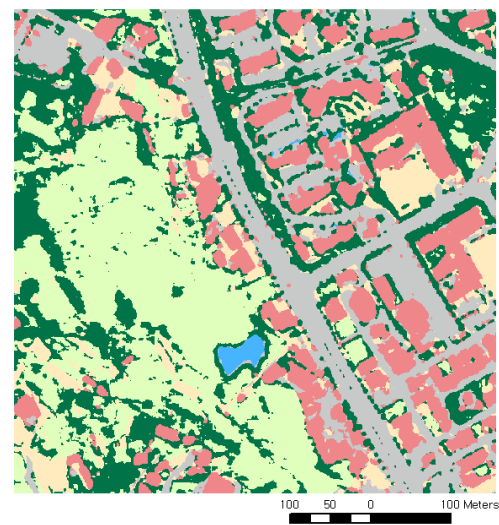

(a)

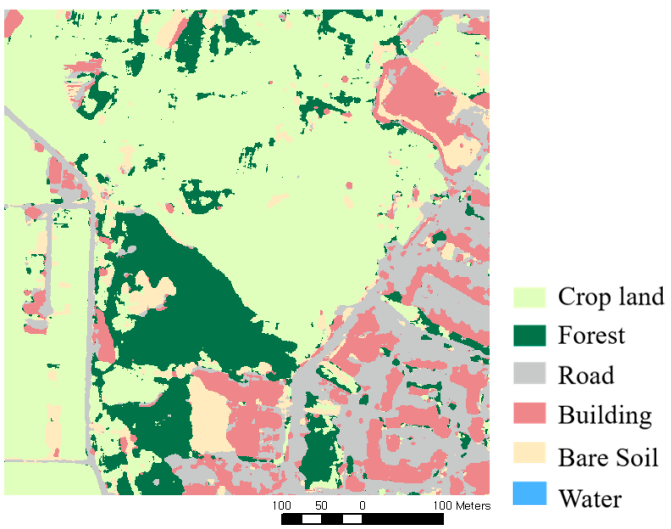

(b)

Figure 8. Classification results of hyperspectral UAV images at the two South Korea sites: (a) Site 1, (b) Site 2 .

\subsection{Detecting Inconsistent Parcels}

According to the relevant regulations in South Korea [21], "cadastral inconsistent parcels" that need to be updated cover the following situations: (1) when the geometric information, such as parcel boundary and area, differs from the actual geometry; (2) when the parcel information is registered incorrectly in the cadastral system; (3) when the parcel information is registered differently from the land survey results; and (4) when the land owner requests an information change. Therefore, we detected the inconsistent parcels requiring update on discrepancies in the land category information.

For the inconsistency comparison, the cadastral maps were converted into a raster structure and compared with the land cover maps generated as the classification results in raster format. The rasterized cadastral map at each site was created by assigning the land category and parcel ID, as shown in Figure 9. The cadastral map comprised polygons containing the parcel boundary information; however, the geometric information representing the parcel boundary was lost during the rasterization process. As a parcel-wise comparison was required for detecting the inconsistent areas between two maps, the cadastral map was rasterized with the parcel ID, as shown in Figure 9b,d. Figure 9 shows the rasterized cadastral map with land category with the same color palette as that of the land cover map, whereas randomly selected colors were used to represent a rasterized cadastral map with parcel ID.

Site 1

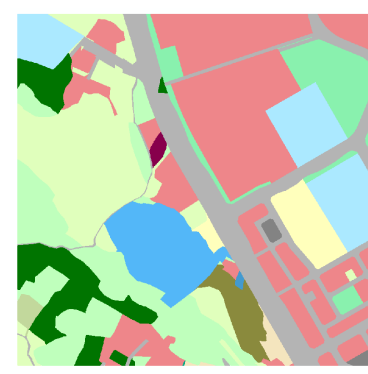

(a)

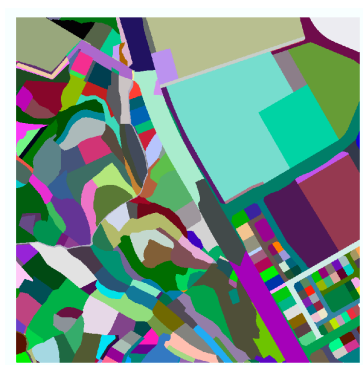

(b)

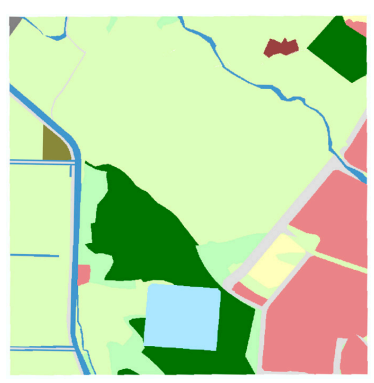

(c)
Site 2

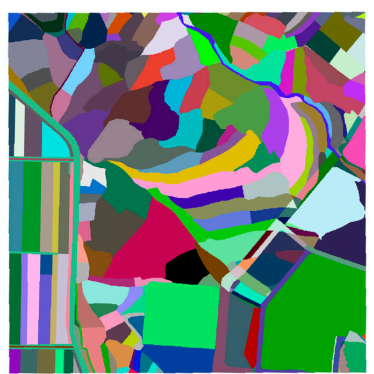

(d)

Figure 9. Cadastral maps rasterized with (a) land category at Site 1, (b) parcel ID at Site 1, (c) land category at Site 2, and (d) parcel ID at Site 2.

The query-based comparison could be automated because each polygon contained the land cover information from the HSI along with the land category information from the cadastral map. Classifying the actual land cover information from images in response to the cadastral map system, specifically to the land category framework, is a technically difficult problem [15]; the criteria for defining the land 
cover classes that can be extracted from HSIs are difficult to reconcile with the land category items in the cadastral map. Therefore, before identifying the inconsistent areas in the cadastral map and the actual land cover map, we must establish a mapping rule between the matched classes under each framework. Then, the consistency of the land cover and land category can be determined from the mapping rule. Because the land category items and land cover classes were classified by different criteria, they could not be matched one-to-one. Based on empirical investigations of the test sites, this study defines M:N matching pairs of land category items and land cover classes (Figure 10). Finally, a query for inconsistency comparison could be made using the mapping rule shown in Figure 10.

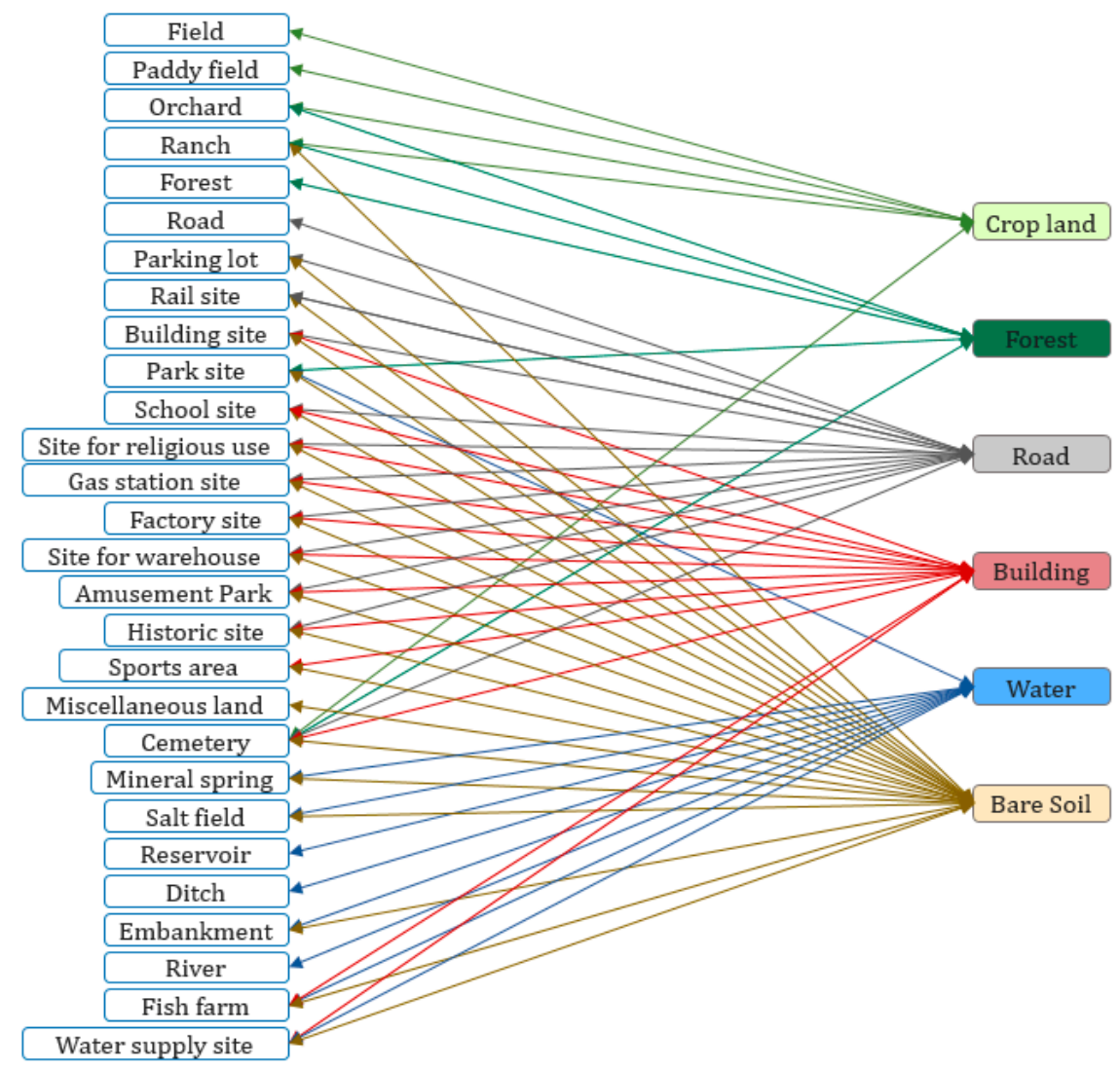

Figure 10. Mapping rule between land category and land cover.

Land cover was classified into crops, forests, buildings, roads, water, and bare soil, which can be distinguished in HSIs. Figure 10 is constructed from the 28 land category items used in the Korean cadastral system. These items and their rules can be adjusted to other cadastral systems, providing source information for other countries. Furthermore, because the query can be modified according to the mapping information, the proposed technique is applicable to the discrepancy analyses of other cadastral systems.

Panels (a) and (d) of Figure 11 present the combined raster maps of Sites 1 and 2, respectively. In these maps, the land cover, land category, and parcel boundary information were combined by encoding with the rasterized cadastral maps and the generated land cover map. The combined raster map was restructured in vector format, and the parcel boundary with the land cover and land category attributes was retrieved by decoding the assigned values (Figure 11b,e; note that each parcel contains many polygons). Finally, the discrepancy map was generated by dissolving the combined vector map based on the parcel ID, leaving only the parcel boundaries, as shown in Figure 11c,f. 


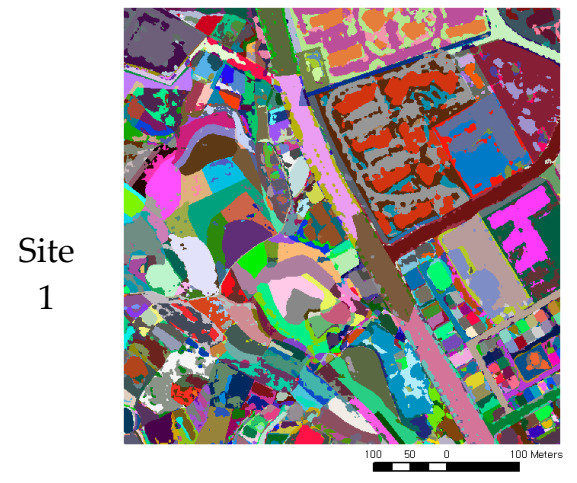

(a)

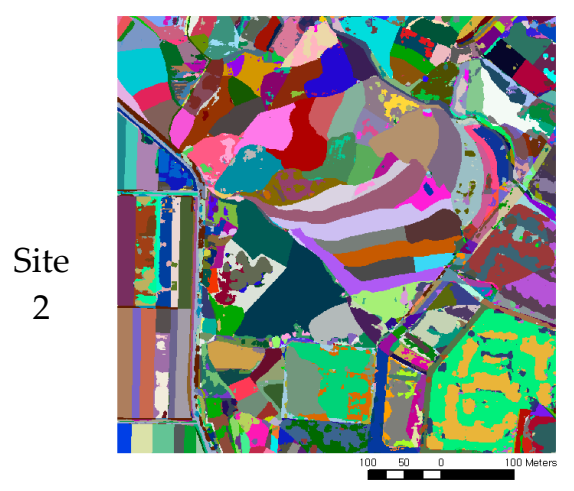

(d)

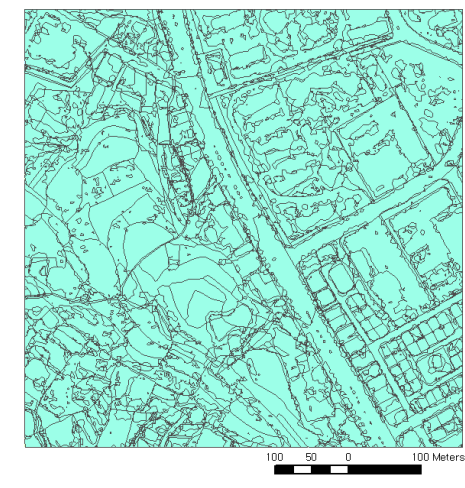

(b)

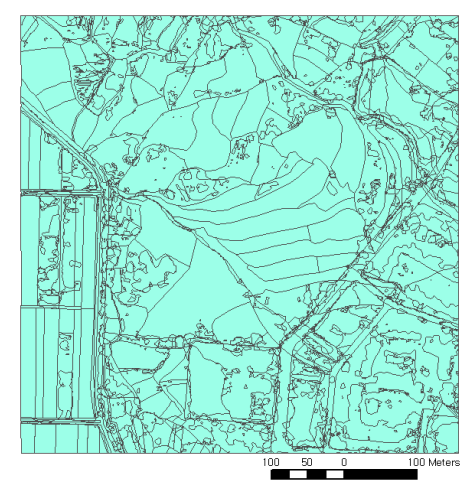

(e)

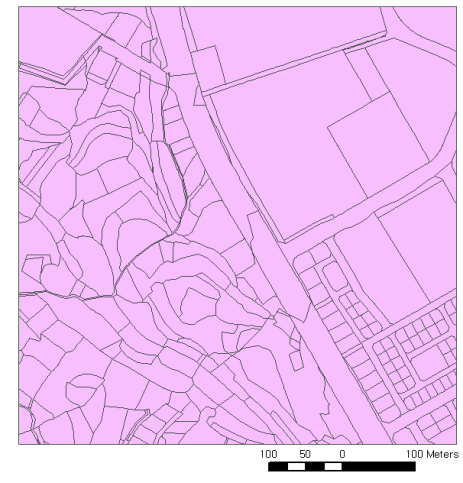

(c)

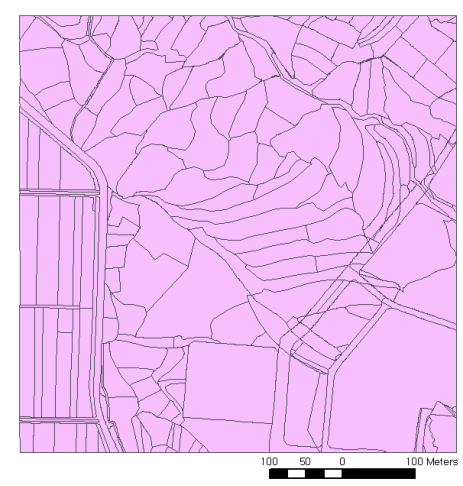

$(\mathbf{f})$

Figure 11. Result of the proposed process: (a) combined raster map of Site 1, (b) combined vector map with attributes of Site 1, (c) discrepancy map of Site 1, (d) combined raster map of Site 2, (e) combined vector map with attributes of Site 2, and (f) discrepancy map of Site 2.

As mentioned earlier, land categories in Korea's cadastral system should be registered based on the primary use of each parcel. The regulations [21] state that if the proportion of parcels used for purposes other than the primary use exceeds a certain level, the parcels must be divided. The polygons in each parcel in the vector layer (Figure 11b,e), which is the intermediate result of the current study, contained the detailed land cover information extracted from the HSI. Therefore, the potential areas to be divided could be concurrently investigated by calculating the polygon area per usage.

The discrepancy maps included the sum of the inconsistent areas as an attribute of each parcel. Therefore, the ratio of the inconsistent area to the total area of each parcel could be calculated. This discrepancy ratio represents the degree of discrepancy (in parcel units) between the land category information registered in the cadastral system and the actual land cover information. Parcels requiring an update of their land category were then identified as those with a large discrepancy ratio. Figure 12 shows the visualization result of the discrepancy ratios between the registered land category information and the actual land cover information extracted from the hyperspectral UAV imagery. If the discrepancy ratio in a parcel exceeds a certain ratio, and the land use of the parcel differs from the registered land category information, this parcel must be separately managed through a site survey. In Figure 12, the discrepancy degree is indicated by a red scale that ranges from white (no discrepancy) to deep red (high discrepancy). In this visualization, the target parcels to be managed can be clearly identified. However, because the threshold discrepancy ratio is not systematically defined, Figure 12 presents three maps of each site with different intervals of discrepancy ratio. More specifically, the inconsistent land parcels in Figure 12c,f are extracted under more rigid criteria than those shown in Figure 12a,d. The deep red regions are the areas that need updating. 


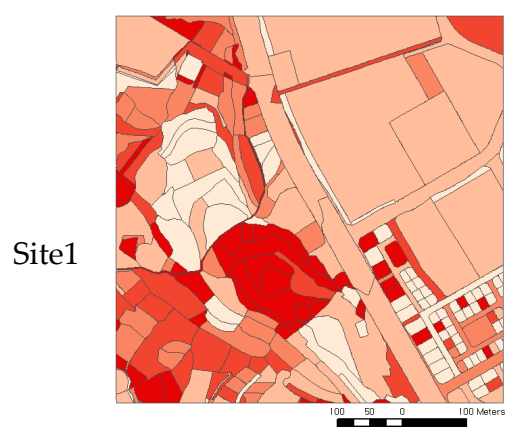

(a)

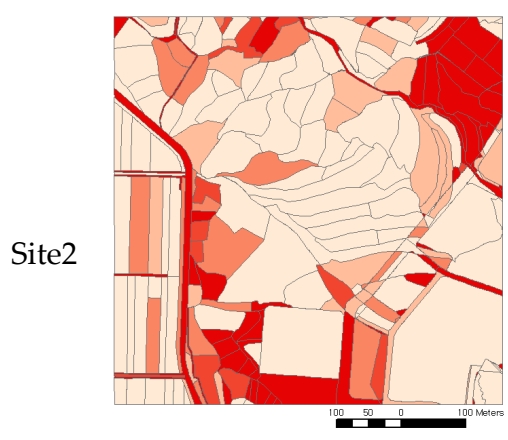

(d)

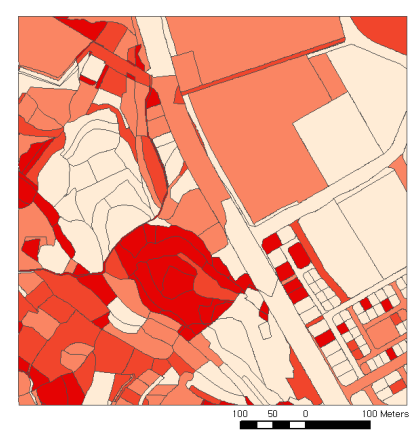

(b)

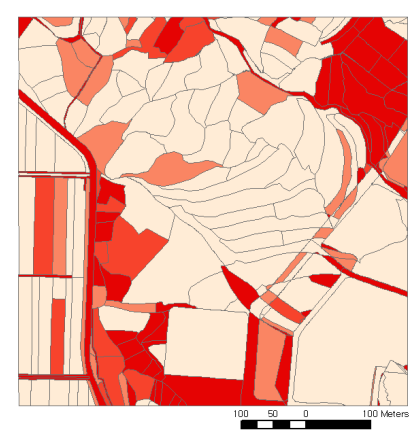

(e)

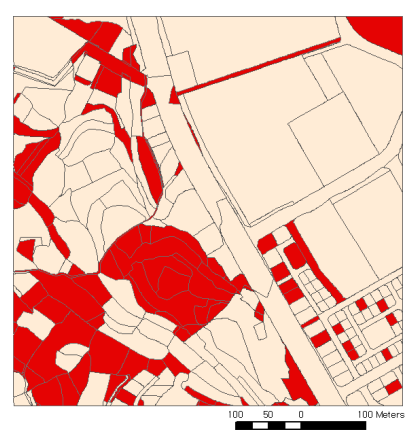

(c)

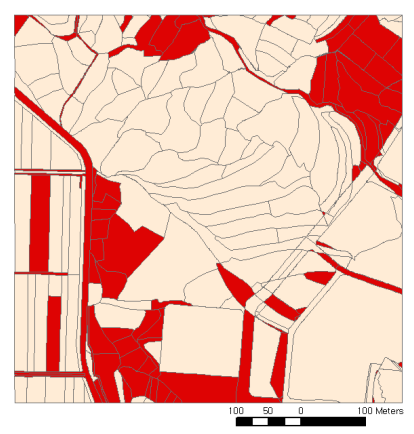

(f)

Figure 12. Visualization result of discrepancy ratios (the intensity of the red polygons directly relates to the discrepancy ratio): (a), (d) with five classes, (b), (e) with four classes, and (c), (f) with two classes.

Table 3 lists the numbers and land types of parcels with discrepancy ratios of $50 \%$ or higher. School sites, cemeteries, and factory sites have relatively low inconsistency probabilities because these land categories were mapped in a 1:N relationship over various land covers. However, paddy fields and bare fields encompass several inconsistent parcels, because many parcels classified as bare soil are actually crop lands that did not bear any crop at the image acquisition time. In particular, significantly fewer discrepancies of building sites and roads were found at Site 2 than at Site 1, because Site 2 covers many fields, paddy fields, and forests, and fewer urban areas such as building sites and roads.

Table 3. Numbers of parcels with discrepancy ratios of $50 \%$ or higher.

\begin{tabular}{cccc}
\hline Land Category & Land Cover & Site 1 & Site 2 \\
\hline Building site & Road, Building, Bare soil & 17 & 0 \\
\hline Paddy Field & Crop land & 24 & 44 \\
\hline Park site & Forest, Water, Bare soil & 0 & 0 \\
\hline School site & Road, Building, Bare soil & 0 & 0 \\
\hline Road & Road & 15 & 2 \\
\hline Field & Crop land & 22 & 25 \\
\hline Forest & Forest & 9 & 7 \\
\hline Cemetery & Road, Building, Bare soil, & 0 & 0 \\
\hline Reservoir & Crop land, Forest & 18 & 0 \\
\hline Miscellaneous land & Water & 2 & 0 \\
\hline Site for Religious use & Road, Building, Bare soil & 0 & 0 \\
\hline Gas station site & Road, Building, Bare soil & 0 & 0 \\
\hline Parking lot & Road, Bare soil & 1 & 1 \\
\hline Sport area & Building, Bare soil & 1 & 0 \\
\hline Ditch & Water & 2 & 7 \\
\hline Factory site & Road, Building, Bare soil & 0 & 0 \\
\hline Ranch & Crop land, Forest, Bare soil & 1 & 0 \\
\hline Total & Bare soil & 112 & 86 \\
\hline
\end{tabular}




\section{Discussion}

\subsection{Analysis of Inconsistent Parcels}

Figures 13 and 14 show the detailed results in two enlarged zones of Site 1 and Site 2, respectively. Some land parcels registered as "building site" on the cadastral system (i.e., designated for building construction) contained no buildings in the real dataset. These parcels often remained as a ground covered only by low vegetation. Thus, the discrepancy ratio was high in parcels of building sites in the cadastral map, but which were classified as crop land or bare soil in subset-1 of Site 1 (Figure 13a). Moreover, it was high in parcels of parking lots in the cadastral map, but identified as buildings in the land cover map. On "road," where the item of the cadastral map exactly matched the class of the land cover, the discrepancy ratios were increased to moderate because the boundaries were sharp on the cadastral map but fuzzy on the land cover map (the roadside trees were included in the road parcels). Although some parcels with more than $50 \%$ of discrepancy ratio were detected as candidates of updating, because of misclassified pixels, they showed a relatively low discrepancy ratio compared to other inconsistent parcels. In subset 2 of Site 1, the discrepancy ratio was high in the central part of the image because many parcels were registered as "reservoir" in the cadastral map but were actually crop land in the land cover map (Figure 13b).

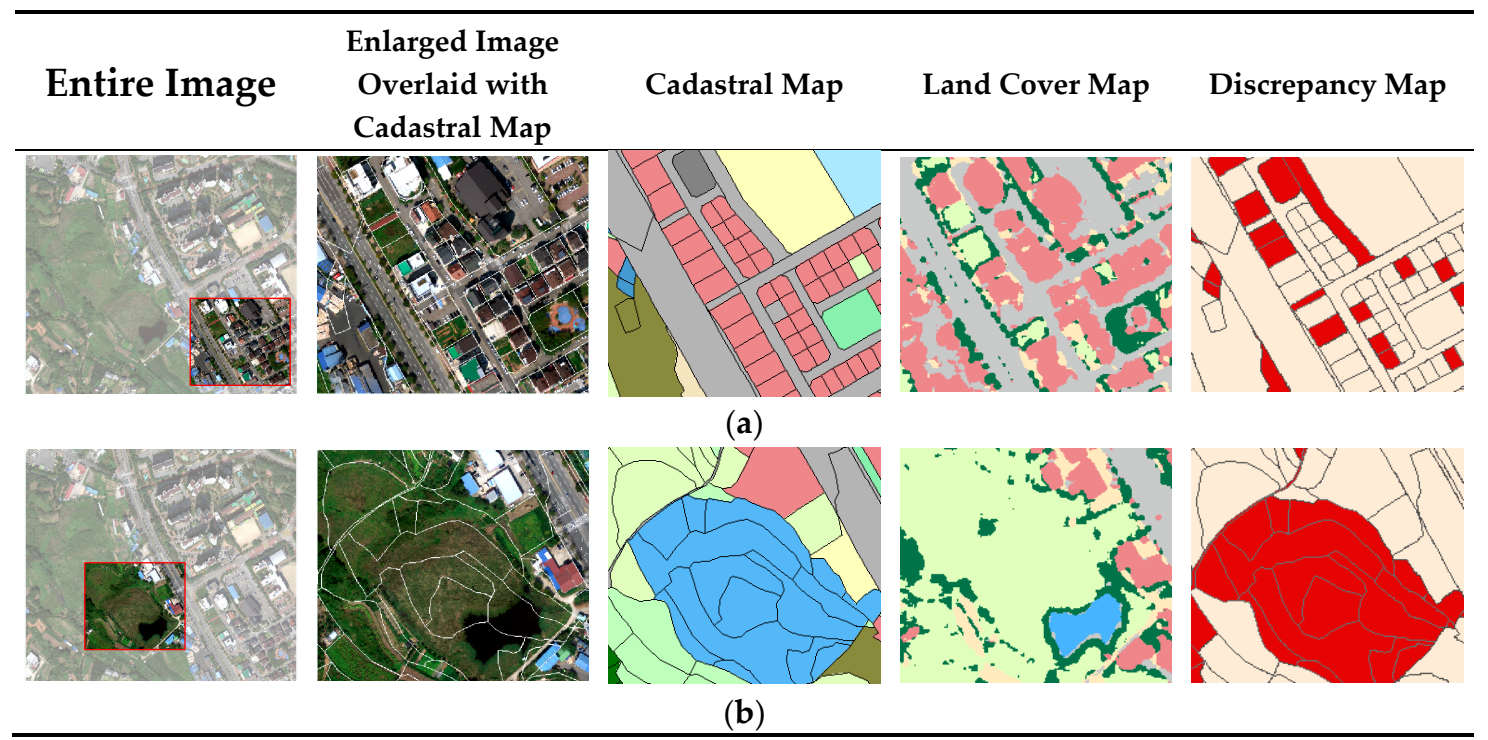

Figure 13. Enlarged hyperspectral UAV images, cadastral maps, land cover maps, and discrepancy maps in two zones of Site 1: (a) subset-1 and (b) subset-2.

In subset 2 of Site 2, some parcels were moderately inconsistent, identified as "paddy field" in the cadastral map, but as bare soil in the land cover map (Figure 14b). In a similar case of subset 1 of Site 2, the discrepancy ratio was high in areas identified as "paddy field" in the cadastral map, but classified as not only bare soil but also buildings in the land cover map. It seems that buildings were constructed on this site (Figure 14a). Moreover, if the vegetation index is low at the image acquisition time, paddy fields can be classified as bare soil. 


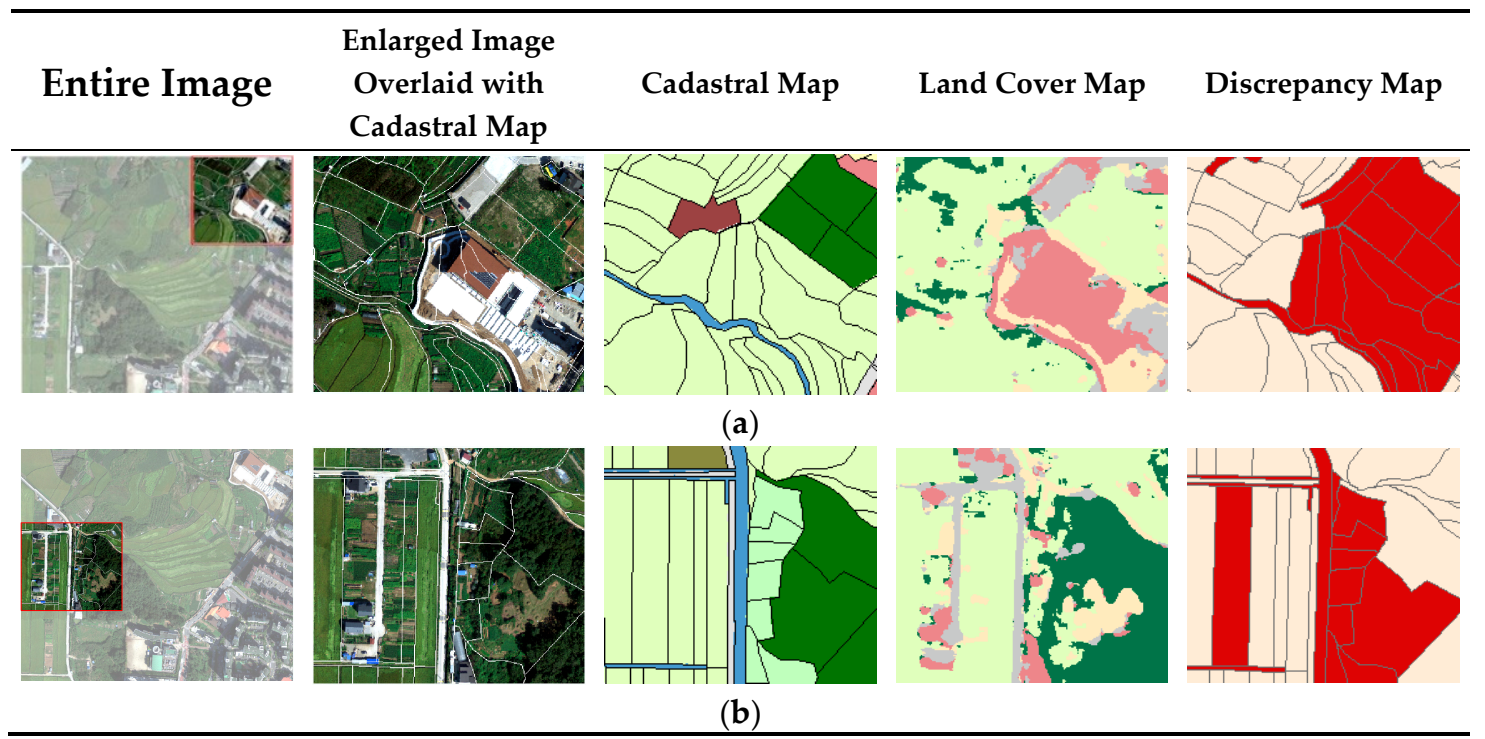

Figure 14. Enlarged hyperspectral UAV images, cadastral maps, land cover maps, and discrepancy maps in two zones of Site 2: (a) subset-1 and (b) subset-2.

\subsection{Limitation and Future Work}

Although the proposed method efficiently extracts inconsistent parcels by an automatic process, its performance depends on the acquisition time of the hyperspectral UAV image, which is input to the discrepancy analysis. For example, some crop parcels are erroneously classified as bare soil because the vegetation vitality is low when the image is captured. The results of the discrepancy analysis may depend on the matching criteria of the land category items and land cover classes, because no absolute rules for defining inconsistency can be established. In conclusion, changing queries for the comparison must be preceded according to the mapping criteria. On the plus side, the proposed method is generalizable to various cadastral systems through flexible modification of the matching criteria between the land categories and land coverage.

In a future, we will develop a classification network that distinguishes finer classes in hyperspectral UAV images with higher classification accuracy. For example, crop land can be divided into rice fields and other fields for finding complex matching relationships between land use and the land categories of cadastral maps.

\section{Conclusions}

Non-spatial data in cadastral maps, such as land use and land ownership is generally updated by field survey and updated manually after visual interpretation of source data, such as RS imagery. This study proposed an approach for analyzing the inconsistent areas between cadastral maps and hyperspectral UAV images. The proposed methods focus on the update of land category which is the attribute data that explain the characteristics of the parcel.

As a case study, the proposed discrepancy analysis was applied to the South Korea cadastral map, which includes 28 land categories. Land cover maps were generated from hyperspectral UAV images by using a hybrid CNN. The hybrid CNN outperformed previous 2D-CNN and 3D-CNN. The OAs of the land cover map using the hybrid CNN at Sites 1 and 2 were $99.93 \%$ and $99.75 \%$, respectively. For comparing the two heterogeneous datasets, the existing cadastral map and the land cover map were encoded. After vectorization, the attributes of the combined vector map were decoded to recover the information of land categories and their coverage. The final discrepancy maps with different discrepancy ratios were generated through a query-based comparison. The discrepancy map reveals the inconsistent parcels, which are used illegally or which need to be subdivided. The 
discrepancy ratios of $39.4 \%$ and $34.4 \%$ of the parcels at Sites 1 and 2, respectively, were $50 \%$ or higher. The discrepancy was high in parcels containing building sites or newly constructed buildings on the cadastral map, but were being used as crop land. As our approach can automate the detection of inconsistent land parcels, it is expected to be applied to large areas and various scenarios. Therefore, they are time- and cost-effective alternatives to field surveys for cadastral map updates and the update cycle can be shortened because the required imagery is taken by UAVs.

Author Contributions: Conceptualization, Methodology, Software, Validation, Formal analysis, Data curation, Writing (original draft preparation), Writing (review and editing), A.S. and S.P.; Funding acquisition, Supervision, Project administration, A.S. All authors have read and agreed to the published version of the manuscript.

Funding: This research was supported by the National Research Foundation of Korea (NRF) funded by the Korean government (MSIT) (grant no. 2019R1A6A3A0109230211) and by a research and development program funded by the Spatial Information Research Institution of Korea Land and Geospatial Informatix Corporation.

Conflicts of Interest: The authors declare no conflict of interest.

\section{References}

1. Kumar, V.G.; Reddy, K.V.; Pratap, D. Updation of cadastral maps using high resolution remotely sensed data. Int. J. Eng. Adv. Technol. 2013, 2, 50-54.

2. Poornima, A.; Jagadeeswaran, R.; Kannan, B.; Sivasamy, R. Generating cadastral base for Kolathupalayam village in Tamil Nadu from high resolution LISS IV sensor data. J. Appl. Nat. Sci. 2016, 8, 2007-2010. [CrossRef]

3. Xia, X.; Persello, C.; Koeva, M. Deep fully convolutional networks for cadastral boundary detection from UAV images. Remote Sens. 2019, 11, 1725. [CrossRef]

4. Pržulj, D.; Radaković, N.; Sladić, D.; Radulović, A.; Govedarica, M. Domain model for cadastral systems with land use component. Surv. Rev. 2019, 51, 135-146. [CrossRef]

5. Ali, Z.; Tuladhar, A.; Zevenbergen, J. An integrated approach for updating cadastral maps in Pakistan using satellite remote sensing data. Int. J. Appl. Earth Obs. 2012, 18, 386-398. [CrossRef]

6. Rao, S.; Sharma, J.; Rajasekhar, S.; Rao, D.; Arepalli, A.; Arora, V.; Kuldeep, C.; Singh, R.; Kanaparthi, M. Assessing usefulness of high-resolution satellite imagery (HRSI) for re-survey of cadastral maps. In Proceedings of the ISPRS Annals of the Photogrammetry, Remote Sensing and Spatial Information Sciences, Hyderabad, India, 9-12 December 2014; Volume 2, pp. 133-143.

7. Jasińska, E.; Preweda, E. Determining the cadastral-tax areas for the real estate premises based on the model of qualitative and quantitative. In Proceedings of the Environmental Engineering 10th International Conference, Vilnius, Lithuania, 27-28 April 2017.

8. Heipke, C.; Woodsford, P.A.; Gerke, M. Updating geospatial databases from images. In ISPRS Congress Book; Taylor \& Francis Grop: London, UK, 2008; pp. 355-362.

9. Koeva, M.; Muneza, M.; Gevaert, C.; Gerke, M.; Nex, F. Using UAVs for map creation and updating. A case study in Rwanda. Surv. Rev. 2018, 50, 312-325. [CrossRef]

10. Crommelinck, S.; Bennett, R.; Gerke, M.; Nex, F.; Yang, M.; Vosselman, G. Review of automatic feature extraction from high-resolution optical sensor data for UAV-based cadastral mapping. Remote Sens. 2016, 8, 689. [CrossRef]

11. Wassie, Y.A.; Koeva, M.N.; Bennett, R.M.; Lemmen, C.H.J. A procedure for semi-automated cadastral boundary feature extraction from high-resolution satellite imagery. J. Spat. Sci. 2018, 63, 75-92. [CrossRef]

12. Avramović, M.; Cvijetinović, Ž.; Mihajlović, D. Digital cadastral map updating status analysis in Serbia. In Proceedings of the International Scientific Conference and XXIV Meeting of Serbian Surveyors 'Professional Practice and Education in Geodesy and Related Fields', Danube, Serbia, 24-26 June 2011.

13. AL-Hameedawi, A.; Mohammed, S.; Thamer, I. Updating cadastral maps using GIS techniques. Eng. Technol. J. 2017, 35, 246-253.

14. Khadanga, G.; Jain, K.; Merugu, S. Use of OBIA for extraction of cadastral parcels. In Proceedings of the International Conference on Advances in Computing, Communications and Informatics (ICACCI), Jaipur, India, 21-24 September 2016.

15. Sung, C.J.; Lim, I.S. A objective study of non-coincidence between land category in cadastral map and land cover classification using satellite images. J. Korean Cadastre Inf. Assoc. 2008, 10, 177-190. 
16. Crommelinck, S.; Bennett, R.; Gerke, M.; Yang, M.; Vosselman, G. Contour detection for UAV-based cadastral mapping. Remote Sens. 2017, 9, 171. [CrossRef]

17. Sim, S.; Song, D. Evaluation of cadastral discrepancy and continuous cadastral mapping in coastal zone using unmanned aerial vehicle. J. Coast. Res. 2018, 85, 1386-1390. [CrossRef]

18. Manyoky, M.; Theiler, P.; Steudler, D.; Eisenbeiss, H. Unmanned aerial vehicle in cadastral applications. In Proceedings of the International Conference on Unmanned Aerial Vehicle in Geomatics (UAV-g), Zurich, Switzerland, 14-16 September 2011.

19. Puniach, E.; Bieda, A.; Ćwiąkała, P.; Kwartnik-Pruc, A.; Parzych, P. Use of unmanned aerial vehicles (UAVs) for updating farmland cadastral data in areas subject to landslides. ISPRS Int. J. Geo-Inf. 2018, 7, 331. [CrossRef]

20. Gajendra, S.; Gopal Narayan, B. An assessment on cadastral map update technologies in Nepal. Crisisonomy 2017, 13, 133-142.

21. Ministry of Land, Infrastructure and Transport. Act on the Establishment, Management, Etc. of Spatial Data; MOLIT: Sejong-si, Korea, 2017.

22. Ministry of Land, Infrastructure and Transport. Act on Land Survey, Waterway Survey and Cadastral Records; MOLIT: Sejong-si, Korea, 2013.

23. Zhang, M.; Li, W.; Du, Q. Diverse region-based CNN for hyperspectral image classification. IEEE Trans. Image Process. 2018, 27, 2623-2634. [CrossRef]

24. Yang, X.; Ye, Y.; Li, X.; Lau, R.Y.; Zhang, X.; Huang, X. Hyperspectral image classification with deep learning models. IEEE Trans. Geosci. Remote Sens. 2018, 56, 5408-5423. [CrossRef]

25. Lee, H.; Kwon, H. Going deeper with contextual CNN for hyperspectral image classification. IEEE Trans. Image Process. 2017, 26, 4843-4855. [CrossRef]

26. Paoletti, M.; Haut, J.; Plaza, J.; Plaza, A. A new deep convolutional neural network for fast hyperspectral image classification. ISPRS J. Photogramm. Remote Sens. 2018, 145, 120-147. [CrossRef]

27. Li, Y.; Zhang, H.; Shen, Q. Spectral-spatial classification of hyperspectral imagery with 3D convolutional neural network. Remote Sens. 2017, 9, 67. [CrossRef]

28. Liu, X.; Sun, Q.; Meng, Y.; Fu, M.; Bourennane, S. Hyperspectral image classification based on parameter-optimized 3D-CNNs combined with transfer learning and virtual samples. Remote Sens. 2018, 10, 1425. [CrossRef]

29. Roy, S.K.; Krishna, G.; Dubey, S.R.; Chaudhuri, B.B. HybridSN: Exploring 3-D-2-D CNN feature hierarchy for hyperspectral image classification. IEEE Geosci. Remote Sens. Lett. 2019, 1-5. [CrossRef]

30. Song, A.; Park, S.; Kim, Y. Updating cadastral maps using deep convolutional network and hyperspectral imaging. In Proceedings of the Asian Conference on Remote Sensing, Daejeon, Korea, 13-18 October 2019.

31. Arcgis Desktop. Available online: http://desktop.arcgis.com/en/arcmap/10.3/analyze/modelbuilder (accessed on 6 December 2019).

32. Arcgis Webmap. Available online: https:/www.arcgis.com/home/webmap/viewer.html?useExisting=1 (accessed on 6 December 2019).

33. Guan, N.; Shan, L.; Yang, C.; Xu, W.; Zhang, M. Delay Compensated Asynchronous Adam Algorithm for Deep Neural Networks. In Proceedings of the IEEE International Symposium on Parallel and Distributed Processing with Applications and 2017 IEEE International Conference on Ubiquitous Computing and Communications (ISPA/IUCC), Guangzhou, China, 12-15 December 2017.

(C) 2020 by the authors. Licensee MDPI, Basel, Switzerland. This article is an open access article distributed under the terms and conditions of the Creative Commons Attribution (CC BY) license (http://creativecommons.org/licenses/by/4.0/). 